\title{
Acquired von Willebrand Syndrome in a Male with Systemic Lupus Erythematosus Presented with Mucocutaneous Bleeding and Treated with rFVIIa
}

\author{
Maryam Hami $^{1}$, Hassan Ahmadnia ${ }^{2}$, Zahra Rezaieyazdi ${ }^{3}$, Hassan Mansouritorghabeh ${ }^{4 *}$ \\ ${ }^{1}$ Kidney Transplantation Complications Research Center, Department of Internal Medicine, Ghaem Hospital, School of Medicine, \\ Mashhad University of Medical Sciences, Mashhad, Iran; ${ }^{2}$ Endoscopic \& Minimally Invasive Surgery Research Center, Ghaem Hos- \\ pital, School of Medicine, Mashhad University of Medical Sciences, Mashhad, Iran; ${ }^{3}$ Rheumatic Disease Research Center, Ghaem \\ Hospital, School of Medicine, Mashhad University of Medical Sciences, Mashhad, Iran; ${ }^{4}$ Allergy Research Center, Ghaem Hospital, \\ School of Medicine, Mashhad University of Medical Sciences, Mashhad, Iran. \\ Email: ${ }^{*}$ Mansouritorghabeh@mums.ac.ir
}

Received August 13 ${ }^{\text {th }}, 2013$; revised September $10^{\text {th }}, 2013$; accepted October $6^{\text {th }}, 2013$

Copyright (c) 2014 Maryam Hami et al. This is an open access article distributed under the Creative Commons Attribution License, which permits unrestricted use, distribution, and reproduction in any medium, provided the original work is properly cited. In accordance of the Creative Commons Attribution License all Copyrights (C 2014 are reserved for SCIRP and the owner of the intellectual property Maryam Hami et al. All Copyright (c) 2014 are guarded by law and by SCIRP as a guardian.

\begin{abstract}
Background: Systemic lupus erythematosis (SLE) is a disorder with multiple organ involvement. Haematological abnormalities have been addressed in it, but acquired von Willebrand syndrome is a rarer phenomenon in current disease. The Case: We report acquired von Willebrand syndrome and SLE in a man with brown rash on face, gingival bleeding, easy bruising and epistaxis and laboratory finding of decreased complement, high level of anti-nuclear antibody and anti-DNA. These findings confirmed the diagnosis of SLE. He underwent kidney biopsy and experienced severe pain at the site of biopsy, but the ultra-sonography evaluation showed small sub capsular haematoma at the site of biopsy. During the next 48 hours, gradually APTT prolongation was continued and haematocrit dropped. In spite of FFP infusion and taking tranexamic acid every eight hours, there wasn't any improvement in haemostatic condition. He received Methylprednisolone and Cyclophosphamid pulses. The patient underwent surgery to roll out vascular complication, but there wasn't any vascular problem. On the third day, recombinant activated factor VII was infused every two hours until oozing was stopped.
\end{abstract}

\section{KEYWORDS}

Acquired von Willebrand Disease; Lupus Erythematosus Systemic; rFVIIa; Bleeding

\section{Introduction}

The von Willebrand disease (vWD) comprises most common inherited bleeding disorder in the world with prevalence about 1\% - 2\% [1]. In 1968, Simon et al. described a case of acquired von Willebrand syndrome (AvWS) in systemic lupus erythematosus (SLE) for the first time [2]. AvWS is less known for clinicians [3] and may occur in association of various underlying disorders including lympho and myeloproliferative disorders, neoplasias, hypothyroidism, Wilms, tumor, congenital heart failure, and thrombocythemia after usage of some drugs

"Corresponding author.
[4]. This plays up on immunologic entity of current syndrome [5]. The current bleeding disorder has absorbed attention during recent years [6]. In comparison with congenital vWD, haemorrhagic manifestations including mucocutanous and gastrointestinal bleeding and post trauma haemorrhage are similar, which are compatible with types I and II vWD [4]. Beside existence of various underlying disorders, the entity of bleeding disorder may be heterogeneous. Also there is no past history of bleeding tendency in bleeder and in his or her family members.

The bone stone of treatment comprises elimination of underlying disease. It is not successful in the case of acute bleeding and necessary invasive procedure [6]. The 
other therapeutic options are infusion of high dose immunoglobulin, plasma exchange, Corticosteroid, Tranexamic acid and recombinant activated Factor VII (rFVIIa).

In the current paper, we present our experience on a case with AvWS in association with SLE. The clinical pictures suggestive of SLE were the first manifestation of disease and AvWS was diagnosed subsequently. The therapeutic regimen including Methylprednisolone (one gr/day IV) $\times$ five and infusion of FFP and tranexamic acid was done without response to bleeding. The surgery for refuting vascular complications and removal of emerged haematoma and overcoming bleeding was done using infusion of rFVIIa successfully.

\section{The Case}

A 25-year-old man was referred to hospital due to epigastic pain, peripheral artheralgia, and lethargy during the last 3 months before admition in hospital. He also complained from gingival bleeding, easy bruising and epistaxis following small trauma. In the physical exam, low grade fever, malar rash, high blood pressure was cleared. Based on current manifestations and laboratory findings (Table 1), SLE was diagnosed. Further laboratory evaluations revealed decreased level of $\mathrm{C}_{3} \& \mathrm{C}_{4}$ and high level of anti-nuclear antibody (ANA) and anti-DNA, and high erythrocyte sedimentation rate (ESR). The urinalysis revealed severe proteinuria ( $3+$ ), mild haematuria $(1+)$, and 24-hour urine evaluation was associated with 2.4 gr/day proteinuria. Based on American Rheumatism Association criteria, these results were compatible with renal involvement in SLE, so on the second day, the patient was candidate for kidney biopsy to recognize its staging. The patient had an APTT test with $45 \mathrm{~s}$ (reference range: 30 - 40 s), so two bags of FFP had been infused to him one hour before biopsy. The kidney's biopsy interpretation was as follow, in light microscopy: diffuse global proliferative and sclerosing lupus nephritis

Table 1. The immunological and haematological parameters of the patient with SLE \& AvWS.

\begin{tabular}{|c|c|c|}
\hline Parameters & Patient & Reference values \\
\hline ANA & 86 & 86 \\
\hline Anti-DNA & 212 & $\leq 50 \mathrm{IU} / \mathrm{ml}$ \\
\hline $\mathrm{C}_{3}$ & 32 & 89 - 187 mg/dl \\
\hline $\mathrm{C}_{4}$ & 8 & $16.5-38 \mathrm{mg} / \mathrm{dl}$ \\
\hline ESR & 46 & $<10 \mathrm{~mm}$ \\
\hline $\mathrm{RBC}$ & $3.22 \times 10^{6} / \mu \mathrm{l}$ & $4.5-5.5$ \\
\hline Hct & $24.5 \%$ & 35 \\
\hline $\mathrm{Hb}$ & $6.2 \mathrm{~g} / \mathrm{dl}$ & $13-17$ \\
\hline Plt & $185 \times 10^{3} / \mu 1$ & $(150-450) \times 10^{3} / \mu \mathrm{l}$ \\
\hline PT & $12.5 \mathrm{~s}$ & $13-15 s$ \\
\hline Activity & $81.5 \%$ & $75-1005$ \\
\hline APTT & $47 \mathrm{~s}$ & $30-40 s$ \\
\hline $\mathrm{APTT}_{\text {mix }}$ & $38 \mathrm{~s}$ & $30-40 s$ \\
\hline Factor VIII inhibitor & Negative & $<0.5$ B. U. \\
\hline $\mathrm{Cr}$ & $3.4 \mathrm{mg} / \mathrm{dl}$ & up to $1.5 \mathrm{mg} / \mathrm{dl}$ \\
\hline \multicolumn{3}{|l|}{ U/A: } \\
\hline Protein & $(3+)$ & Negative \\
\hline Blood & $(4+)$ & Negative \\
\hline Proteinuria & 2.4 gr/day & Negative \\
\hline WBC & Many & $0-2$ \\
\hline Crystal & Amorphus urate $(+4)$ & Negative \\
\hline
\end{tabular}

ANA: anti-nuclear antibody; Anti DNA: anti-deoxy nucleic acid; IU: international unit; dl: deci liter; mm: mili-meter; ESR: erythrocyte sedimentation rate; C: complement; WBC: white blood cell; RBC: red blood cell; Hct: haematocrit; Hb: haemoglobin; Plt: platelet; PT: prothrombin time; INR: international normalized ratio; APTT: activated partial thromboplastin time; S: second; $\mu$ : micro-liter; Cr: cratinin; U/A: urine analysis; gr: gram. 
and membranous lupus nephritis compatible with lupus nephritis class $\mathrm{V}$, activity index 14 and chronicity index two was reported. Immuno-flourescent (IF) microscopy showed glomerular staining for IgG, IgA, IgM, C1q, C3, Kappa and Lambda. The IF staining results were similar in all of them, diffuse granular pattern in the glomerular basement membrane (GBM).

The patient experienced pain in his left of abdomen at site of biopsy after that. The ultra sonography evaluation showed sub capsular haematoma at place. As a result of disease activity, he received one gram daily Methylprednisolone pulse for five days and one gram Cyclophosphamid pulse. In following days further haemostatic evaluation showed APTT: 55 s (reference range 30 - 40 s), PT: $12.5 \mathrm{~s}$ (reference range $13 \mathrm{~s}$ ), platelet count $185 \times$ $10^{3} / \mu 1$ and mixed APTT: $38 \mathrm{~s}$. The existence of factor VIII inhibitor excluded by detection of factor VIII inhibitor using Bethesda assay. He received FFP $(20 \mathrm{ml} / \mathrm{kg})$, three bags/day and Tranexamic acid every eight hours for the upcoming 48 hours. Despite of these efforts, blood oozing continued from biopsy place and sub capsular hematoma was growing. So due to low level of hemoglobin ( $\mathrm{Hb}: 6.5 \mathrm{mg} / \mathrm{dl}$ ), two packed red blood cell was transfused. The renewed APTT showed elevation of APTT to $96 \mathrm{~s}$ and second sonography showed enlargement of haematoma. The patient underwent for surgery to roll out vascular complication as a result of kidney biopsy, but there wasn't any vascular problem. Administration of rFVIIa began for him with dosage of 90 micro$\mathrm{gram} / \mathrm{kg}$ every two hours for two days and then every six hours until oozing of site of cutting occurred. This regimen continued until blood oozing from the wound of surgery was stopped.

Repeated sonography showed no recurrent haematoma. Administration of high dose intravenous steroid began for him, subsequently followed by prescription of oral prednisolone. In follow up, in the next months, he received 6 dose of Cyclophosphamid monthly. In sixth month after treatment, laboratory data revealed normal renal function and 3+ proteinuria (4.07 gr/24 hours). We didn't visit him till six months later, when he didn't take any drugs. Blood pressure began to rise so we recommended to him to take Losartan, Hydrochlorotiazid, Mycophenolate Mofetil and Prednisolon. Unfortunately after a month, he denied to take his drugs. After one year he came back to clinic, at this time he only took $5 \mathrm{mg}$ Prednisolone and the lab test revealed normal renal function and 1+ proteinuria. Although hypertension became aggravated, Amlodipine was added to his drug regimen. He did not come to our clinic during next year, after coming to clinic in the next year, he claimed that has refused to take Prednisolone and antihypertensive drugs from 10 months ago. Lab data at that time showed normal renal functions and urine was negative for proteinu- ria. Only hypertension was remained.

\section{Discussion}

The bleeding tendency in the patient described above was typical for acquired von Willebrand syndrome rather than inherited. First, the patient had not any haemorrhagic tendency until the age of 25 years old. He even passed circumcision without abnormal bleeding. Second, there was no family history of bleeding disorders. Third, the pattern of bleeding manifestation as mucosal bleeding was consistent with AvWS. Fourth, unresponsive of haemorrhage to infusion of cryoprecipitate or FFP was compatible with AvWS.

The autoantibody directed at vWF/FVIII can make complex that clear from circulation speedily. This is the reason for poor responses to treatment with FVIII concentrate, vWF/FVIII concentrate, desmopressin acetate and cryoprecipitate in some types of AvWS. It has been reported that administration of high-dose intravenous immunoglobulin (IVIG), and elevation of plasma levels of both vWF and FVIII are seen in AvWS associated with SLE transiently [4].

Treatment policy comprised successful management of underlying disease that may lead to disappearance of haemorrhagic tendency secondary to AvWS in some cases. The main therapeutic approaches for control of bleeding in AvWS are administration of desmopressin acetate (that is effective in about $30 \%$ of AvWS) and FVIII/vWF concentrate. The third therapeutic option is high dose administration of IVIG (one g/kg for two days) $[3,4]$. There are other therapeutic options that comprise rFVIIa [7], plasma exchange [8], Corticosteroids [9] and Tranexamic acid [10]. Returning plasma levels of factor VIII and vWF to reference levels after prednisone therapy are indicative of immune entity of AvWS in SLE [4].

The presented clinical manifestations, laboratory data, type of bleeding and clinical course in current case indicate that he was a rare case of AvWS in association with SLE. We did a search on reported cases using AvWS keyword in Pub Med search engine and limitation of English language literature (Table 2). We could only find five papers who presented eight cases. The review of bleeding manifestations showed among nine various bleeding symptoms that have been reported, epistaxis (seven episodes) and easy bruising (four episodes) were the commonest. Among various clinical signs, the commonest were pain of joints (three episodes) and fatigue (three episodes). All of reported cases revealed haemorrhage before presentation of SLE, while in current cases haemorrhagic picture began concomitant with SLE pictures.

A number of mechanisms have been suggested for AvWS including reduced synthesis and formation of inhibitor against vWF. In the current case the synthesis of 
Table 2. Summary of published haemorrhagic manifestations of AWS cases with SLE.

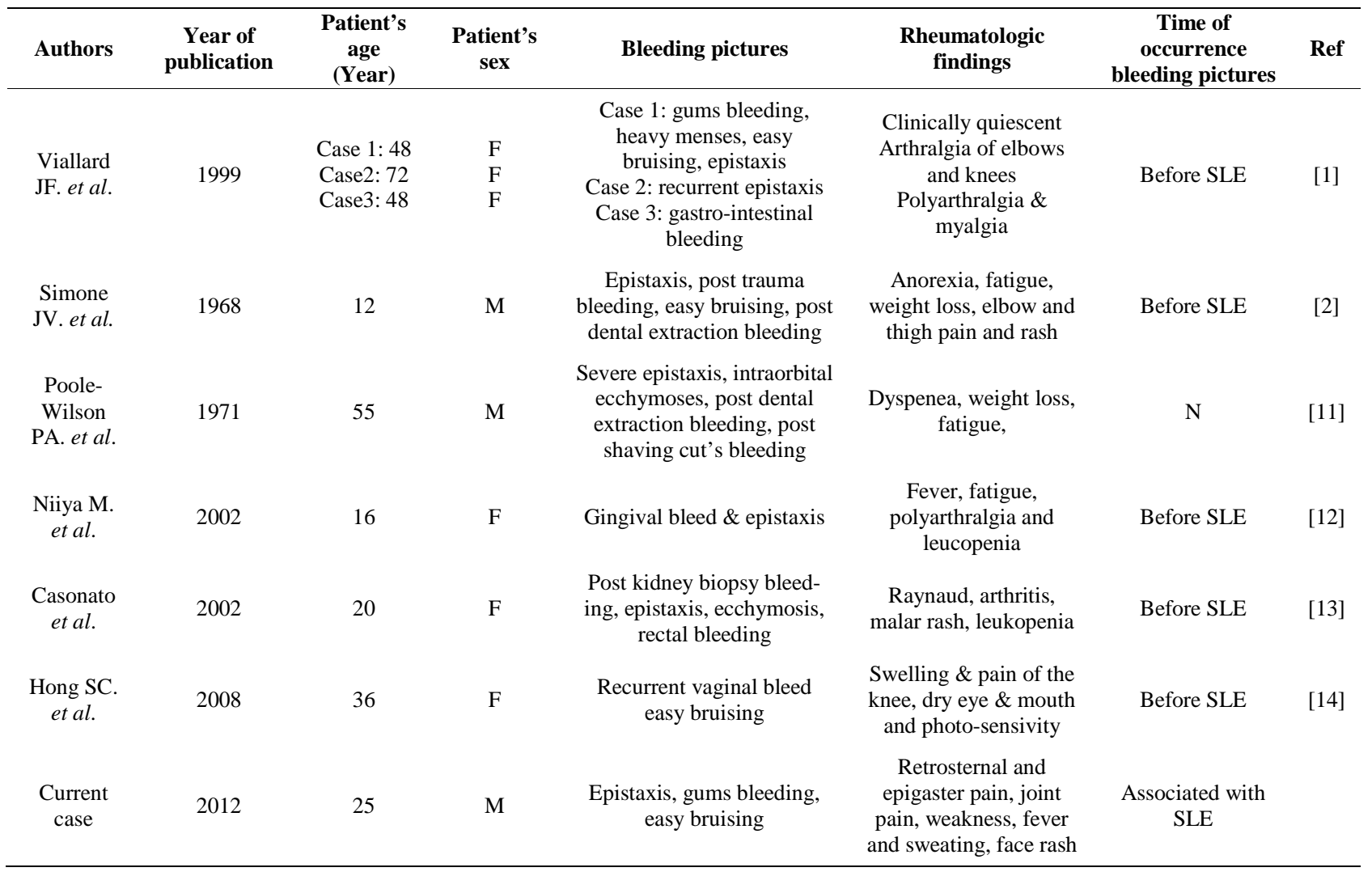

F: female; M: male; N: not reported; SLE: systemic lupus erythematosus; Ref: reference.

vWF should be impaired because both mixing text (equal volume of patient's plasma and pooled plasma) and Bethesda assay were negative. Lack of haemostsis laboratory equipped with aggregometry is a necessity that does not exist in our region. It causes problems both for diagnose and treatment of patients. In similar condition diagnosis can be established on clinical evidences.

\section{Conclusion}

In conclusion, based on current limited data on haemorrhagic episode secondary to underlying disease and post invasive procedure haemorrhage, in cases with AvWS invasive procedures and limited surgeries may be efficiently and successfully treated and managed with rFVIIa, provided that a multidisciplinary team works together to establish extra care in covering haemostasis challenges and close observation.

\section{Conflict of Interest}

There is no conflict of interest to poising the manuscript.

\section{Acknowledgements}

The authors would like to thank Dr. Reza Hekmat for referring current case for further evaluations and establishing treatment.

\section{REFERENCES}

[1] J. F. Viallard, J. L. Pellegrin, C. Vergnes, A. Borel-Derlon, G. Clofent-Sanchez and A. T. Nurden, "Three Cases of Acquired von Willebrand Disease Associated with Systemic Lupus Erythematosus,” British Journal of Haematology, Vol. 105, No. 2, 1999, pp. 532-537. http://dx.doi.org/10.1111/j.1365-2141.1999.01360.x

[2] J. V. Simone, J. A. Cornet and C. F. Abildgaard, “Acquired von Willebrand Syndrome in Systemic Lupus Erythematosus,” Blood, Vol. 31, No. 6, 1968, pp. 806812.

[3] H. Mohri, “Acquired von Willebrand Syndrome: Features and Management," American Journal of Hematology, Vol. 81, No. 8, 2006, pp. 616-623. http://dx.doi.org/10.1002/ajh.20455

[4] J. J. Michiels, W. Schroyens, M. van der Planken and Z. Berneman, “Acquired von Willebrand Syndrome in Systemic Lupus Erythematosus," Clinical and Applied Thrombosis and Hemostasis, Vol. 7, No. 2, 2001, pp. 106-112. http://dx.doi.org/10.1177/107602960100700205

[5] M. R. Rinder, R. E. Richard and H. M. Rinder, "Acquired von Willebrand Disease: A Concise Review,” American 
Journal of Hematology, Vol. 54, No. 2, 1997, pp. 139145.

http://dx.doi.org/10.1002/(SICI)1096-8652(199702)54:2< 139::AID-AJH7>3.0.CO;2-Y

[6] H. Mohri, "Acquired von Willebrand Syndrome: Its Pathophysiology, Laboratory Features and Management," Journal of Thrombosis and Thrombolysis, Vol. 15, No. 3, 2003, pp. 141-149.

http://dx.doi.org/10.1023/B:THRO.0000011369.70824.e6

[7] P. W. Friederich, P. C. Wever, E. Briet, C. J. Doorenbos and M. Levi, "Successful Treatment with Recombinant Factor VIIa of Therapy-Resistant Severe Bleeding in Patient with Acquired von Willebrand Disease," American Journal of Hematology, Vol. 66, No. 4, 2001, pp. 292294. http://dx.doi.org/10.1002/ajh.1060

[8] L. E. Silberstein, J. Abrahm and S. J. Shattil, "The Efficacy of Intensive Plasma Exchange in Acquired von Willebrand's Disease,” Transfusion, Vol. 27, No. 3, 1987, pp. 234-237.

http://dx.doi.org/10.1046/j.1537-2995.1987.27387235627 $\underline{\underline{X}}$

[9] A. K. Stewart and M. F. Glynn, "Acquired von Willebrand's Disease Associated with Free Lambda Light Chain Monoclonal Gammopathy, Normal Bleeding Time and Response to Prednisone," Postgraduate Medical Journal, Vol. 66, No. 777, 1990, pp. 560-562. http://dx.doi.org/10.1136/pgmj.66.777.560
[10] J. C. J. Eikenboom, F. J. M. van der Meer and E. Briet, "Acquired von Willebrand's Disease Due to Excessive Fibrinolysis," British Journal of Haematology, Vol. 81, No. 4, 1992, pp. 618-620.

http://dx.doi.org/10.1111/j.1365-2141.1992.tb03005.x

[11] P. A. Poole-Wilson, “Acquired von Willebrand's Syndrome and Systemic Lupus Erythematosus," Proceedings of the Royal Society of Medicine, Vol. 65, No. 6, 1972, pp. 561-562.

[12] M. Niiya, K. Niiya, Y. Takazawa, Y. Hayashi, M. Y. Tanio, M. Kushiro, M. Tanimizu, H. Hasegawa and M. Tanimoto, “Acquired Type 3-Like von Willebrand Syndrome Preceded Full-Blown Systemic Lupus Erythematosus,” Blood Coagulation and Fibrinolysis, Vol. 13, No. 4, 2002, pp. 361-365. http://dx.doi.org/10.1097/00001721-200206000-00013

[13] A. Casonato, E. Pontara, A. Doria, A. Bertomoro, M. G. Cattini, P. F. Gambari and A. Girolami, "Lack of Multimer Organization of von Willebrand Factor in an Acquired von Willebrand Syndrome," British Journal of Haematology, Vol. 116, No. 4, 2002, pp. 899-904. http://dx.doi.org/10.1046/j.0007-1048.2002.03341.x

[14] S. Hong, J. Lee, H. Chi H, C. Lee, S. Nah, Y. G. Kim, J. S. Oh, H. B. Moon and B. Yoo, "Systemic Lupus Erythematosus Complicated by Acquired von Willebrand's Syndrome,” Lupus, Vol. 17, No. 9, 2008, pp. 846-848. http://dx.doi.org/10.1177/0961203308089429 